\title{
Transoral robotic surgery in dilated cardiomyopathy patients: Anaesthetic considerations
}

\author{
Anita Kulkarni, Uma Hariharan* and Ajay Kumar Bhargava \\ Rajiv Gandhi Cancer Institute and Research Centre, Rohini, New Delhi, India
}

\begin{abstract}
Dilated cardiomyopathy $(\mathrm{DCM})$ is a serious cardiac condition resulting in progressive heart failure and death. There have been several reports of anaesthetic management of DCM patients for various surgeries. Trans-Oral Robotic Surgery (TORS) is a minimally invasive approach to radical surgery of head and neck cancers. It utilizes the da Vinci ${ }^{\mathrm{TM}}$ robotic system to excise deep-seated intra-oral tumors in difficult to reach areas. We present a case series of 3 patients with DCM for TORS and their anesthetic management.
\end{abstract}

\section{Introduction}

The WHO (World Health Organization) classifies cardiomyopathies according to the anatomical and physiological features into 3 main types: dilated, hypertrophic and restrictive. In dilated cardiomyopathy (DCM), the major pathophysiology is systolic dysfunction with global reduction in myocardial contractility. After an initial enlargement of the left ventricle, the compensatory mechanisms reach a nadir, causing left ventricular failure and death. As the low cardiac output state progresses, there is generalized edema, valvular regurgitation, rhythm disturbances and sometimes, intra-cardiac thrombus. Medical management consists of ACE (Angiotensin-Converting Enzyme) Inhibitors, diuretics, beta-blockers, digoxin and anticoagulants. Some patients require biventricular pacing (coordinated stimulation of both the ventricles, improving the stroke volume). Transoral robotic surgery (TORS) is a minimally-invasive approach to deep-seated head and neck cancerous lesions, using the daVinci ${ }^{\mathrm{TM}}$ robotic surgical system (Intuitive Surgical, Sunnyvale, CA, USA) [1]. TORS has several advantages, in the form of less blood loss, better cosmesis, minimal postoperative pain and early recovery. We hereby present a case series of three patients with DCM posted for TORS and the anesthetic challenges faced.

\section{Case reports}

All three patients posted for TORS were already diagnosed cases of DCM. A thorough pre-operative evaluation was done, with explanation of risks, possible postoperative retention of endotracheal tube and intensive care unit (ICU) stay. Pre-anaesthetic investigations included routine hemogram, serum electrolytes, coagulation profile, chest x-ray, electrocardiogram, renal and liver function tests. All patients also underwent a cardiac evaluation and echocardiographic analysis for ventricular function, valvular status, possibility of thrombus and regional wall motion abnormalities. If patient was on ACE (angiotensin-converting enzyme) inhibitors or angiotensin receptor blockers, they were omitted on the day of surgery to prevent sudden hypotension on induction. Beta-blockers and digoxin were continued. None of the patients were on anticoagulants or pacers. Sedative premedicants in the ward were avoided to prevent myocardial depression or airway obstruction. All preparations for difficult airway management were done, including fibreoptic bronchoscope, videolaryngoscope, cricothyroidotomy and tracheostomy. Adequate nasal preparations were carried out and the more patent nostril was selected for nasotracheal intubation, after preoxygenation. In view of the associated cardiac condition and major cancer surgery, all three patients underwent placement of invasive monitoring lines (arterial and central venous catheters) pre-induction under local anaesthesia, with ultrasound guidance. Apart from standard ASA (American Society of Anesthesiologists) monitors (ECG, NIBP, $\mathrm{SpO}_{2}, \mathrm{ETCO}_{2}$, Temperature), neuromuscular monitoring, bispectral index, multigasmonitor, arterial blood pressure, central venous pressure, cuff pressures and urine output were monitored in all patients. Postoperatively, the patients were reversed, but not extubated, in anticipation of airway edema following intra-oral surgery. Flexomettalic tube was changed to portex (PVC) cuffed endotracheal tube (ETT) with the help of a tube exchanger device before reversal. All patients were monitored in a dedicated onco-surgical intensive care unit with the nasotracheal tube in-situ. All of them received humidified oxygen through a T-piece. These patients were extubated after 24 hours over an airway exchange catheter, with supplemental esmolol boluses in-order to prevent sympathetic stimulation. Postoperative analgesia was multimodal, with intravenous paracetamol, NSAID`s (non-steroidal antiinflammatory agents) and Fentanyl infusion (30-40 mcg/hour) for the first 24 hours. One patient developed arrhythmia post-extubation, which was amenable to medical management. There were no other complications in any patient.

Correspondence to: Hariharan U, MBBS, DNB, PGDHM, FellowOncoanesthesia, Rajiv Gandhi Cancer Institute and Research Centre, Rohini, New Delhi, India; Currently, Specialist- Anaesthesia and Intensive Care, Delhi Government Health Services, New Delhi, India, Tel: +919811271093; E-mail: uma1708@gmail.com

Key words: dilated cardiomyopathy, transoral robotic surgery, cardiac failure, davinci robot

Received: September 12, 2015; Accepted: October 23, 2015; Published: October 28,2015 


\section{Case 1}

A 60 year old, $62 \mathrm{Kg}$, hypertensive, male patient with DCM and growth on posterior aspect of the tongue was posted for TORS. He was a reformed smoker and alcoholic. There was no history of atrial fibrillation or endocarditis. On chest X-ray, there was cardiomegaly and ECG (electrocardiogram) showed occasional missed beats. Echocardigraphy showed global hypokinesia, reduced LV (left ventricular) systolic function with septal wall motion abnormality, mildly dilated LA (left atrium) and left ventricular ejection fraction $($ LVEF $)=35 \%$. He was on oral tablets (Tab.) Torsemide $10 \mathrm{mg}$ BD (twice daily), Tab. Carvedilol $12.5 \mathrm{mg}$ OD (once daily), Tab. Telmisartan $40 \mathrm{mg}$ BD and Tab. Rosuvastatin $10 \mathrm{mg}$ OD. After applying all monitors (including invasive lines), anesthesia was induced with intravenous (I.V) Midazolam (1mg), Fentanyl (100 mcg), Morphine $(6 \mathrm{mg})$ and Etomidate $(8 \mathrm{mg})$. C-MAC ${ }^{\mathrm{TM}}$ videolaryngoscope was used for guiding the nasotracheal tube into the vocal cords. I.V Esmolol bolus $(60 \mathrm{mg}$ ) followed by infusion were given to suppress the sympathetic response during the insertion of mouth gag and robotic instruments. Anesthesia was maintained with $\mathrm{O} 2+$ Air+Desflurane. Patient maintained his vital parameters (Pulse, blood pressure, Airway pressure, CVP) within normal limits throughout. Esmolol infusion was continued till the removal of mouth-gag and then tapered off. After the surgery, the patient was reversed, but not extubated. Flexomettalic tube was changed to cuffed PVC (polyvinyl chloride) tube and he was transferred to the ICU (intensive care unit) for overnight retention of tube and continued monitoring. He was extubated the next day over a tube exchanger device and then shifted to the ward after 48 hours. All his preoperative cardiac medicines were continued and the patient was asked to regularly follow-up with the cardiologist and the oncosurgeon.

\section{Case 2}

A 59 year old, $66 \mathrm{Kg}$ male patient diagnosed to have carcinoma of left base of tongue with DCM was posted for TORS. He was a chronic smoker, with history of palpitations and hypertension. He had been hospitalized 6 months back for cardiac failure and was currently on the following medications: Tab Telmisartan $40 \mathrm{mg} \mathrm{BD}$, Tab Eplerenone (aldosterone receptor antagonists) $25 \mathrm{mg}$ OD and Tab Carvedilol (selective beta blocker) $6.25 \mathrm{mg}$ OD. All blood investigations were within normal limits. Chest X-ray showed cardiomegaly and ECG showed occasional ectopics. Echocardiography showed severe global hypokinesia, paradoxical septal wall motion abnormality, reduced systolic function, grade 2 diastolic dysfunction, dilated LV cavity.and $\mathrm{LVEF}=34 \%$. Preoperative vitals were: Pulse $80 / \mathrm{min}$, B.P $120 / 82 \mathrm{mmHg}$ and $\mathrm{SpO} 2$ 98\%. Invasive monitoring was instituted along with routine monitors. After preoxygenation, I.V induction with midazolam $2 \mathrm{mg}$, Fentanyl $120 \mathrm{mcg}$, Morphine $6 \mathrm{mg}$ and Etomidate $10 \mathrm{mg}$ was done. For attenuation of intubation response, I.V Esmolol bolus was given @ $1 \mathrm{mg} / \mathrm{Kg}$. A flexomettalic tube was inserted nasotracheally under videolaryngoscopic guidance. Muscle relaxant given after confirming tube position and continuous vecuronium infusion started. Anesthesia was maintained with $\mathrm{O} 2+\mathrm{Air}+$ Sevoflurane. During surgery, heart rate was maintained between $68-82 / \mathrm{min}$, BP range $110-140$ systolic/70-80 diastolic mmHg, central venous pressure (CVP) 6-9 cms of $\mathrm{H} 2 \mathrm{O}$ and urine output $40 \mathrm{ml} /$ hour. In order to attenuate the sympathetic stimulation caused by mouth gag and insertion of robotic instruments into the oral cavity, Esmolol infusion @ $0.3 \mathrm{mg} / \mathrm{Kg} / \mathrm{min}$ was started and continued till removal of mouth gag. After de-docking, nasogastric tube was inserted through the other nostril and a softened portex (PVC) tube railroaded over a tube exchanger device. Patient was not extubated and shifted to the ICU for observation and monitoring. Postoperative analgesia was with I.V paracetamol and continuous morphine infusion@ @ - 3 mg/hour. He was extubated the next day over an airway exchange catheter. The rest of the perioperative course was uneventful.

\section{Case 3}

A 40 year old, $70 \mathrm{Kg}$, male patient with hypertension and DCM was posted for TORS. He had history of chronic tobacco intake. His blood investigations were normal. Chest Xray revealed cardiomegaly. ECG showed $\mathrm{T}$ inversion in antero-lateral leads. Echocardiography revealed severely reduced LV systolic function, global hypokinesia, mild MVP (mitral valve prolapse), trivial MR/TR (mitral/tricuspid regurgitation) and $\mathrm{LVEF}=28 \%$. He had mild trismus, reduced mouth opening and Mallampati grade 3 on airway assessment. Awake-FOB guided nasotrachaeal intubation was planned after upper airway anesthesia, preoxygenation and mild I.V sedation (Midazolam $1 \mathrm{mg}+$ Fentanyl $20 \mathrm{mcg}$ ). To attenuate the response to intubation, continuous Esmolol infusion (@15 mg/min) was started along with invasive monitoring. I.V Etomidate $(12 \mathrm{mg}$ ) and Atracurium bolus $(30 \mathrm{mg})$, followed by infusion $(30 \mathrm{mg} /$ hour$)$ was given after confirming the tube position. I.V Fentanyl $(80 \mathrm{mcg})$ and Morphine $(4.5 \mathrm{mg})$ were given for analgesia. Anesthesia was maintained with $\mathrm{O} 2+\mathrm{Air}+$ Sevoflurane. Nitroglycerin (NTG) infusion (5-7 $\mathrm{mcg} / \mathrm{min}$ ) was used for controlling the pressor response to insertion of mouth gag and robotic instruments. CVP was maintained between $7-10 \mathrm{cms}$ of $\mathrm{H} 2 \mathrm{O}$ and fluid restriction. As in the previous cases, invasive monitoring, overnight retention of tube in ICU and extubation after 24 hours over a tube exchanger device was practiced in this patient also. Post-extubation, he developed rthythm disturbances in the form of atrial fibrillation, which was hemodynamically stable. Pharmacological cardioversion was done with I.V Amiodarone (Bolus dose $150 \mathrm{mg}$ diluted in $100 \mathrm{ml}$ of $5 \%$ dextrose over 10 minutes followed by infusion of $1 \mathrm{mg} / \mathrm{min}$ over next 6 hours and $540 \mathrm{mg}$ over the remaining 18 hours). The patient responded well and reverted to sinus rhythm. He was observed in the ICU for 72 hours with expert consultation from a cardiologist. Patient remained hemodynamically stable throughout and was later shifted out on oral cardiac medications.

\section{Discussion}

Dilated cardiomyopathy is a serious cardiac disorder causing progressive life-threatening cardiac failure [2]. All three patients were already diagnosed cases of DCM with concurrent deep-seated intraoral cancer, posted for minimally-invasive robotic surgery for excision. They were medically managed for DCM under a cardiologist care, with no evidence of cardiac failure or arrhythmias or intra-cardiac thrombus. In elective cases, medical control of cardiac failure for $>1$ week is recommended. Treatment of arrhythmias, particularly atrial fibrillation and correction of electrolyte derangements must be done preoperatively. All their blood investigations were normal. In view of anticipated difficult airway, the videolaryngoscope was used in the first two patients for securing the airway and muscle relaxant given only after confirming correct tube position [3]. Nasotracheal intubation was done to give enough room for the surgeon and his robotic instruments. Flexomettalic tube was selected for intubation so as to prevent tube kinking or occlusion by the robotic arms and mouth gag. TORS is a promising, minimally invasive head and neck robotic surgery, with the ability to work around corners, while avoiding certain line-ofsite limitations. Awake FOB-guided nasal intubation was employed in the last patient in view of limited mouth opening and trismus [4]. 
Etomidate was used for intravenous induction due to its cardiovascular stability. Muscle relaxant infusion under neuromuscular monitoring was employed to maintain adequate surgical relaxation and prevent even slight patient movement during robotic surgery. Intra-operative hemodynamic stability was ensured with either esmolol bolus or NTG infusion. The main concern was the rise in sympathetic stimulation following application of mouth gag to open the mouth for accommodating robotic instruments [5]. Laryngeal reflexes can be stimulated by the handling of the areas in-and-around the deepseated cancerous growth. Tachycardia and hypertension can be deleterious in DCM patients and arrhythmias can be stimulated during tumor manipulation, both leading to precipitation of cardiac failure. Invasive monitoring was instituted pre-induction, for beat-to-beat monitoring of the blood pressure, pulse contour analysis and central venous pressure. Complete preparation of the operating room (O.R). with defibrillator, all cardiac medications, resuscitative equipment and pacemaker need to be done preoperatively to effectively manage any untoward events. Difficult airway cart also need to be kept ready in such patients for effective airway management as per ASA difficult airway guidelines. Postoperatively, these patients were not extubated, so as to give adequate time for airway edema to settle [6]. Extubation was done over a tube exchanger device in view of pre-existing difficult airway. Only one patient developed postoperative complication, in the form of atrial fibrillation post-extubation. This could have been because of sympathetic stimulation during the extubation process, transient airway obstruction following tube removal or pre-existing compromised cardiac status (echocardiographic findings of severely reduced systolic function, low ejection fraction, global hypokinesia). Nevertheless, he returned to sinus rhythm following amiodarone and other supportive therapy. The rest of the perioperative course was uneventful in all the patients. They were discharged later with advice for regular follow-up with the cardiologist, onco-surgeon and the oncologist.

\section{Conclusions}

Robotic surgery is a new feather in the revolutionary cap of minimally-invasive surgery. Trans-oral robotic surgery is a promising technique for head and neck cancers in difficult to reach areas. The prime concern is sympathetic stimulation following insertion of mouth gag and robotic arms or instruments during TORS. Other considerations include judicious fluid therapy, cautious positioning and postoperative tube retention, apart from meticulous difficult airway management. A patient with compromised cardiac status can pose a huge challenge for anesthetic management of such complex surgeries. Dilated cardiomyopathy can cause cardiac failure or intractable arrhythmias, especially under the stress of anesthesia and cancer surgery. Detailed preoperative evaluation and adequate preanesthetic preparation, along with institution of invasive monitoring is indispensable for a successful outcome. Anesthetic management must be tailor-made according to individual patient requirements. Intensive post-operative monitoring must be done to observe for development of rhythm disturbances or cardiac failure or thrombo-embolism. Anesthesiologists must be prepared to face the challenges posed by improvements in surgical technology in such high risk cardiac patients. TORS can be safely done in well controlled DCM patients, provided all the above precautions are strictly adhered-to.

\section{References}

1. O'Malley BW Jr, Weinstein GS, Sneider W, Hockstein NG (2006)Transoral robotic surgery (TORS) for base of tongue neoplasms. Laryngoscope116: 1465-1472. [Crossref]

2. Narang S (2007) Anesthetic management of dilated cardiomyopathy. MEJ Anesth 19:243-250. [Crossref]

3. Aziz MF, Dillman D, Fu R, Brambrink AM (2012) Comparative effectiveness of the $\mathrm{C}-\mathrm{MAC}$ video laryngoscope versus direct laryngoscopy in the setting of the predicted difficult airway. Anesthesiology 116: 629-636. [Crossref]

4. Chi JJ, Mandel JE, Weinstein GS, O`Malley BW Jr (2010) Anesthetic conmsiderations for transoral robotic surgery. Anesthesiol Clin 28: 411-422. [Crossref]

5. Narayanaswamy S, Khanna P, Bhalla AP, Singh AK (2012) Perioperative concern in trans-oral robotic surgery: Initial experience of four cases. $J$ Anaesthesiol Clin Pharmacol 28: 226-229. [Crossref]

6. Jeffery LA, Carin AH, Robert AC (2013) Practice guidelines for management of the difficult airway: An Updated Report by the American Society of Anesthesiologists Task Force on Management of the Difficult Airway. Anesthesiology 118: 1-20. [Crossref]

Copyright: (C2015 Kulkarni A. This is an open-access article distributed under the terms of the Creative Commons Attribution License, which permits unrestricted use, distribution, and reproduction in any medium, provided the original author and source are credited. 